\title{
La literatura negroafricana en el marco del comparatismo literario
}

\author{
Nelson Sindze Wembe \\ Universitat de Lleida \\ Papson20@hotmail.com
}

RESUM

\section{La literatura negroafricana en el marc del comparatisme literari}

El present article estudia la relació existent entre la literatura negroafricana escrita i la literatura comparada. Estructurat en dos apartats, la primera part de la nostra anàlisi presenta com ha anat evolucionant el comparatisme literari des de l'eurocentrisme fins a l'obertura a relacions entre la literatura i les altres arts. La segona part exposa les particularitats que la literatura negroafricana aporta a l'ambició del comparatisme que pretén aproximar-se a l'humanisme. Els conceptes d'interculturalitat, transnacionalisme, hibridisme cultural i literari que marquen la creació literària subsahariana són elements importants que el comparatisme literari pot incorporar per concretar la idea de Weltliteratur que l'anima.

Paraules claus

Comparatisme literari, literatura negroafricana, hibridisme, interculturalitat.

\section{RESUMEN}

\section{La literatura negroafricana en el marco del comparatismo literario}

El presente artículo estudia la relación existente entre la literatura negroafricana escrita en lenguas occidentales y la literatura comparada. Estructurado en dos apartados, el primero presenta la evolución del comparatismo literario desde el eurocentrismo hasta la apertura a relaciones entre la literatura y otras artes. La segunda parte se centra en la exposición de las particularidades estéticas, estilísticas y temáticas que la literatura subsahariana aporta a la ambición del comparatismo impulsándola a aproximarse al humanismo. Conceptos como la interculturalidad, el transnacionalismo, el hibridismo cultural y literario que marcan la creación literaria en el sur del Sahara son elementos importantes que el comparatismo puede incorporar para concretar su idea de Weltliteratur.

Palabras claves

Comparatismo literario, literatura negroafricana, hibridismo, interculturalidad.

\section{RÉSUMÉ}

\section{La littérature négroafricaine dans le cadre du comparatisme littéraire}

Le présent article étudie la relation existante entre la littérature négroafricaine écrite en langues occidentales et la littérature comparée. Divisé en deux parties, la première étudie comment le comparatisme littéraire a évolué de l'eurocentrisme à une ouverture de relations entre la littérature et les autres arts. La deuxième partie se centre sur la présentation des particularités esthétiques, stylistiques et thématiques que l'art littéraire négroafricain apporte à l'ambition d'expansion et 
d'humanisme du comparatisme. Les concepts comme l'interculturalité, le transnationalisme, l'hybridisme culturel et littéraire qui marquent la production littéraire négroafricaine sont des éléments importants qu'incorpore le comparatisme littéraire dans la réalisation de sa vision de Weltliteratur.

\section{MoTS CLÉS}

Comparatisme littéraire, littérature négroafricaine, hybridisme, interculturalité.

\section{Abstract}

\section{Black African literature in the framework of literary comparatism}

This article focuses on the study of the existing relationship between black African literature written in western languages and comparative literature. Divided into two parts, the first one shows how literary comparatism has evolved from eurocentrism to the exploration of relationships between literature and other forms of art. The second part presents the esthetical, stylistic and thematic particularities that black African literature provides to the ambition of comparatism by approaching it to humanism. Concepts such as interculturality, transnationalism, cultural and literary hybridism, which leave a mark on sub-Saharan literary production, are important elements with which comparatism constructs the idea of Weltliteratur.

\section{KEYwORDS}

Literary comparatism theory, Black African literature, hybridism, interculturality.

\section{Comparatismo literario: definición y evolución}

Según Pedro Aullón de Haro el comparatismo literario ante todo es una epistemología de crítica previa. Es decir que está asociado "al fenómeno de hacer patente la propia constitución de la obra literaria y la probabilidad de acceso a la misma como objeto crítico bien constituido" ${ }^{1}$. Presentado de esa manera, el crítico establece una superioridad del comparatismo literario para con las demás teorías de crítica literaria. Esa afirmación encuentra su justificación en la esencia de la creación literaria: la necesidad de divulgar una cultura, una creencia o un pensamiento. Para descodificar esos signos socio-culturales dejados consciente o inconscientemente por el autor en su texto, es necesario por ejemplo, establecer las líneas entre lo real y lo ficticio. La comparación se presenta como elemento de base para desmarcar el real de lo ficticio. Sin embargo, se ha de mencionar que como disciplina crítica, como aproximación científica al texto literario, el comparatismo establece sus bases teóricas posteriormente a las críticas literarias generales.

La literatura comparada es una disciplina académica y una teoría de crítica literaria reciente. Esa juventud justifica las constantes expansiones que conoce la disciplina para circunscribir el material de estudio. Dicha adaptación y expansión se verifica en las distintas definiciones que han acompañado su evolución. De un "estudio de las relaciones binarias entre dos literatura o dos elementos

1. Pedro Aullón de Haro, "Epistemología de la teoría y crítica literatura" in Teoría de la crítica literaria, Trotta, Madrid, 1994 p. 22. 
de dos literaturas" ${ }^{2}$, hemos pasado hoy a una definición más transversal y transdisciplinaria. Susana Gil-Albarellos Pérez-Pedrero resalta que:

La literatura comparada se basa principalmente en el análisis de la literatura desde un punto de vista amplio, es decir, con perspectiva multidisciplinar, supranacional y sobre todo paracultural, si es que se nos permite el uso de tal término para referirnos a un campo de estudio que partiendo de la literatura abarca todas las manifestaciones de la cultura ${ }^{3}$.

La transcendencia de la que habla Susana Gil-Albarellos y sobre todo la relación estrecha entre literatura y cultura al que alude la crítica había sido formulada en le pregunta previa que Yves Chevrel (1989) daba al principio del hecho comparatista:

Au point de départ il y'a cette question fondamentale, qui distingue sans doute la littérature comparée des autres disciplines " comparées » : que se passe-t-il quand une conscience humaine, intégrée dans une culture (dans sa culture), est confrontée à une œuvre expression et partie prenante d'une autre culture $?^{4}$

La misión del comparatista es hacer interactuar las culturas y por ello, todo lector diferente a la cultura del autor es un comparatista a su nivel. El fundamento del estudio comparatista según Chevrel es el extranjero, la otredad; ese elemento o esos elementos literarios diferentes que llaman la atención del comparatista. El Otro o el Extranjero aquí se refiere a la vez a ese desconocido que ha escrito y que pertenece a una cultura ${ }^{5}$ y a esos elementos dejados aquí y allá consciente o inconscientemente por el autor y que proporcionan informaciones sobre su personalidad, sus posiciones ideológicas y sociológicas.

La configuración actual de las relaciones y movimientos humanos favorecidos por los progresos científicos y técnicos participan en la caída de las nociones de etnias, comunidad, naciones y favorece la construcción de las nociones de transculturalidad, transnacionalidad, transdisciplinariedad ${ }^{6}$. Si consideramos la sociedad como base de producción literaria, se explica el hecho de que la literatura actual tenga una visión supranacional y un interés universal. El postmodernismo literario es la materialización de la progresiva desaparición de las literaturas nacionales en beneficio de las creaciones literarias transnacionales. Esas mutaciones sociales, políticas y artísticas confieren a la literatura comparada suficientes materiales de expansión. Por eso, desde 1961 con Henri Remark, la literatura comparada ha ido superando el estudio de los encuentros entre literaturas para abrirse

2. Paul Van Tieghem, La littérature comparée, Armand Colin, Paris, 1931.

3. Susana Gil-Albarellos Pérez-Pedrero, Introducción a la literatura comparada, Valladolid: Universidad de Valladolid, Secretariado de publicaciones e Intercambio Editorial, Valladolid, 2006. p. 13.

4. Yves Chevrel, La littérature comparée, Presses Universitaires de France, Paris, 1989. p. 8.

5. Pensamos que es necesario mencionar aquí que la cultura ya sólo no hace referencia a lo que es particular a un conjunto sino al comportamiento del conjunto en un eje sincrónico. Será por ejemplo interesante para el comparatista de ver a través de la literatura la diferencia entre la sociedad negroafricana bajo la colonización y la actual.

6. Con la mundialización y la necesidad casi impuesta de abertura de fronteras, la manifestación de la estrecha relación entre las naciones se ha transferido a casi todos los sectores sociales generando los términos que mencionamos aquí. En general, se trata de una divulgación de valores universales que va más allá de las culturas y las disciplinas o de las naciones para buscar elementos asociados con el humanismo y el universalismo. 
a las relaciones entre la literatura y los demás órganos sociales y científicos. La definición de René Welleck (1970) precisa ese cambio de rumbo en la disciplina:

El estudio de toda la literatura desde una perspectiva internacional. En esta concepción, (...) la literatura comparada es idéntica al estudio de la literatura independientemente de las fronteras lingüísticas, étnicas y políticas. No puede confinarse a un único método ni puede limitarse a los contactos históricos ${ }^{7}$.

Chevrel (1989) en su definición de la literatura comparada aconseja al comparatista no imponerse límites sobretodo lingüísticos. Esa necesitad de libertad a la hora de investigar es la que ha causado seguramente hasta hoy la inestabilidad de los paradigmas comparatistas. Marius-François Guyard advertía que la expansión de la literatura comparada podría causar su perdida: "Je crains fort qu’à vouloir être tout le comparatisme ne soit plus rien"s.

Consideramos el comparatismo como punto de partida del humanismo. Es decir $-\mathrm{y}$ partiendo de lo presentado hasta aquí- el texto literario es sólo elemento base; referente con el cual el comparatista trabaja e investiga la sociedad de su tiempo o de los tiempos pasados. Es la fuente que permite al crítico comprender la creación literaria clásica, la moderna y la postmoderna observando los intercambios entre dichas etapas (estudio de las influencias y de la recepciones). Más allá del texto, el comparatismo arma el comparatista (lector o científico) a poner en comunicación culturas, disciplinas externas a la literaria y comprenderla. Puede que sea por eso que Yves Chevrel menciona que "si le comparatisme a une ambition, c'est d'essayer de contribuer à une forme d'humanisme, qui accorde du prix à toute expression de l'esprit humain ${ }^{\text {x) }}$ basándose en una metodología que privilegia un movimiento de tipo análisis/síntesis/análisis ${ }^{10} \ldots$ Al umbral de las definiciones que hemos mencionado anteriormente, la literatura comparada establece puentes - donde antes no existían - entre culturas, disciplinas, obras y hasta entre el autor y su obra. Su propósito es de crear mayor contacto entre los elementos de la literatura y de la humanidad. Basándonos en lo expuesto, definimos la literatura comparada como una epistemología de crítica previa que toma como base la literatura para relacionarla con distintos dimensiones sociales, culturales, políticas, lingüísticas y humanistas.

Aunque la literatura comparada quiere ser universal, es con displicencia que ha evolucionado de estudios eurocentristas hacia una apertura a otras literaturas nacionales y continentales. El eurocentrismo literario del comparatismo se justifica por el nacimiento de la teoría sobre el continente europeo. Posteriormente, se extiende a Estados Unidos y de ahí también la apertura a las literaturas postcoloniales.

Los estudios postcoloniales se focalizan sobre las producciones de autores provenientes de las antiguas colonias siguiendo una línea de escritura temporal pero también estilística para desmarcar el hibridismo cultural producto de los contactos coloniales. El contacto entre la literatura del

7. Réné Welleck, "the name and nature of comparative literature" en Discrimination, further concepts of criticism, Yale University Press, New Haven and London, 1970, pp. 19-20.

8. Marius-François Guyard, la Littérature comparée, Presses Universitaires de France, Paris, 1978. p. 6.

9. Yves Chevrel (1989); op cit. P.123.

10. Esa metodología científica que según Yves Chevrel (1989) constituye la base de las operaciones científica es fundamental para el comparatismo. Permite al comparatista cuestionar sus síntesis para encontrar nuevos elementos de análisis y no aceptar un resultado como una totalidad sino como punto de partida de otros análisis. 
colonizador, su cultura y la literatura de los colonizados así como su cultura es material importante para la expansión de los estudios comparatistas. Una de las importancias de la interpretación postcolonial es el estudio de las obras de autores, de sus descendientes de familias de inmigrantes y de la experiencia de ésos ${ }^{11}$. Esos escritores de la inmigración o de la diáspora, verdaderos seres híbridos — porque pertenecientes a dos culturas — son los que están mejor situados para hablar de las realidades de sus dos mundos sin tomar partido y por lo mismo responder a las exigencias de los conceptos de Mundialización y Globalización. Jean Marc Moura subraya al respecto que:

\footnotetext{
Par ailleurs, les médias de masse présentent un ensemble de représentations des cultures du monde, à travers lesquelles nous sont livrés des stéréotypes globaux, chargés de résumer de manière emblématique les diverses modalités culturelles. Internet, la télévision et les médias nous transforment en voyageurs globaux consommateurs de clichés. Il semble que ce soit aussi la tâche de la littérature, singulièrement des lettres europhones, de réagir à ceux-ci ou au moins d'en déjouer les faux-semblants. ${ }^{12}$
}

Aunque en los estudios comparatistas actuales estan muy presente las literaturas de Asia, América Latina y del Norte, se nota una escasez de investigaciones comparatistas sobre África subsahariana. Natalia Álvarez Méndez expone que:

$\mathrm{Al}$ margen de las introducciones generales a la literatura comparada y los estudio monográficos sobre la misma, si se revisa la bibliografía comparatista se observará la atención prestada a los diferentes ámbitos europeos, americanos e incluso asiáticos, pero también que África es la gran ausente de las obras programáticas de la disciplina ${ }^{13}$.

Dicha carencia, según Jacques Chevrier, se debe a la juventud de la creación literaria subsahariana. Una juventud que no impide que la literatura negroafricana en particular (por ser la más joven) y la literatura africana en general sean dotadas de elementos que pueden proporcionar al comparatista fuentes para un estudio denso. Además, las literaturas africanas escritas en lenguas europeas tienen una originalidad que brota en los hábitos y actitudes literarias propios del africanismo tradicional ${ }^{14}$. El comparatista sin embargo no sólo se ha de acercar al texto para: "encontrar la africanidad de tal o cual texto cuanto de considerarlo el surgimiento de una consciencia arraigada hic et nunc"15 si no para encontrar los lazos que unen las literaturas negroafricanas entre ellas y dichas literaturas con otras literaturas del mundo. Queda claro entonces que la literatura africana $-y$ la negroafricana en el caso de nuestra investigación - tiene mucho que aportar al comparatismo aunque sea solamente sobre la base de los contactos interculturales e interlingüísticos entre África y los países colonizadores.

11. John McLeod, Beginning postcolonialism, Manchester University Press, Manchester, 2000.

12. Jean Marc Moura, "Postcolonialisme et comparatisme" en Vox poética, 2006, consultable en línea en : http://www. vox-poetica.com/sflgc/biblio/moura.html. (última consulta: 31/1072014).

13. Natalia Álvaro Méndez, "Africanismo y literatura comparada" en Metodologías comparatistas y literatura comparada, Clásico y Yekinson, Madrid, 2012.

14. Hacemos referencia aquí a las costumbres implantadas por movimientos como el panafricanismo, la Négritude, la "African personality".

15. Jacques Chevrier, "Las literaturas africanas en campo de la investigación comparada" en Compendio de literatura comparada (dir: Pierre Brunel; Yves Chevrel), Siglo Veintiuno, Madrid, 1994. p. 189. 


\section{Literatura negroafricana y comparatismo literario}

El enriquecimiento literario del que goza la literatura comparada actualmente se debe sobre todo a su expansión como venimos diciendo. Con los estudios postcoloniales, el comparatismo literario se ha abierto a otras literaturas y por lo mismo ha integrado nuevos elementos de comparación. En los estudios postcoloniales, el hibridismo tanto cultural como literario parece ser el elemento de comparación de predilección por el cual el crítico entra a explorar las obras. El contacto entre las lenguas, entre la cultura del colonizador y del colonizado, entre la literatura de ambas partes son algunas de las líneas explorables. Al lado de esas relaciones, se estudia también el comportamiento de la literatura de los colonizados antes, durante y después de la colonización poniendo en paralelos las particularidades de cada época. Hemos mencionado más arriba que la literatura negroafricana está menos estudiada por los comparatistas pero hay académicos que han publicado sobre el tema ${ }^{16}$. Pensamos - y es lo que expondremos a continuación- que la literatura negroafricana está dotada de componentes susceptibles de enriquecer aún más el comparatismo literario. Entre otros elementos tenemos: la oralidad literaria, el hibridismo literario o cultural, el problema de identidad, los paralelismos tradición-modernidad, la inmigración negroafricana, los conceptos de nacionalidad y etnicidad.

\section{La oralidad}

La oralidad tiene un papel importante en la construcción de la originalidad de la literatura subsahariana. Es a partir de ella y sobre todo de su encuentro con las literaturas europeas que nace lo que venimos llamando la literatura negroafricana escrita o moderna. La literatura oral negroafricana no ha tenido mucha divulgación primero por ser sólo oral pero también por enfrentarse a la diversidad lingüística del continente. La tradición oral de África Negra engloba cuentos, fábulas, proverbios y cantos. Los cuentos o fábulas contados por el Mbomo Mvet (en el centro ecuatorial africano), el griot (en gran parte del Sahel) o las abuelas se trasmitían de generación en generación y permitía exaltar la vida de un rey, de un antepasado. La particularidad de esos géneros es la presencia de los animales como personajes. En cuanto a los proverbios y refranes, o son frutos del mensaje promulgado por un cuento o son metáforas situacionales que permiten dar una advertencia o un consejo.

El trabajo del comparatista consistiría en destacar las similitudes entre los cuentos negroafricanos propios a cada etnia. Se podría también establecer los paralelismos entre otras tradiciones orales (occidentales, asiáticas...). El estudio en ese caso siguiendo las pautas del estructuralismo literario resaltará los parecidos estilísticos, estéticos y semánticos entre los cuentos... Una comparación entre la literatura oral y la literatura escrita negroafricana pueden ser del apego del comparatista. Se sabe por ejemplo que Ahmadou Hampate Bâ publicó una obra bilingüe en peul y francés en la que se recogía cuentos peul (Contes initiatiques peuls; 1994). Birago Diop también es autor de les contes

16. Apoyándose sobre las investigaciones científicas en Francia y Estados Unidos, se puede decir que el campo negroafricano pena en ser estudiado en España. Sin embargo se ha de apreciar proyectos de investigación y algunas tesis doctorales que trazan el camino hacia dicho campo. Durante nuestra investigación hemos computado seis tesis doctorales en español sobre la literatura negroafricana y dos proyectos de investigación (MIGRA: proyecto de constitución de una base de datos de autores inmigrantes en Espańa llevado por la universidad de Santiago de Compostela y el proyecto Afroeuropeos similar a MIGRA pero centrado fundamentalmente sobre autores negroafricanos en Europa llevado a cabo por la Universidad de León). 
d'Ahmadou Koumba y de les nouveaux contes d'Ahmadou Koumba. Sin olvidar los numerosos libros sobre Chaka Zulu, Beyanzin, Soundjata Keita, Pokou que además de ser memorias históricas son obras inspiradas en epopeyas orales negroafricana. El hibridismo literario es la resultante de ese encuentro entre la literatura subsahariana oral y la escrita.

\section{Hibridismo literario}

Alfonso de Toro estructura de forma siguiente la estrategia de hibridación:

La estrategia de hibridación apunta a la potencialización de la diferencia y no a su reducción, asimilación, adaptación, en un primer momento. En un segundo momento conduce la estrategia hibridación a un "reconocimiento de la diferencia", esto es, a la posibilidad de negociar identidades diferentes en un tercer espacio. ${ }^{17}$

El proceso de hibridismo que conoció África fue diferente. Se asistió en muchas colonias (sobre todo francesa, españolas y portuguesas) a una reducción de las culturas locales para una potencialización de las culturas coloniales. Sin embargo, es del contacto colonial entre África y Europa que viene el hibridismo literario negroafricano actual. Entre la criollización del francés (y de las demás lenguas coloniales) de Césaire y el mestizaje senghoriano, la producción literaria negroafricana escrita ha sabido mezclar la tradición oral con los estilos occidentales. African Unbound: Study in Race Emancipation, Les trois volontés de Malik, Cuando los combes luchaban ${ }^{18}$, dan a conocer la cultura negroafricana usando una lengua diferente de las lenguas maternas de los autores. El hibridismo literario aquí tiene un objetivo fundamental: una propaganda de las culturas subsaharianas para hacer frente a los textos escritos por misioneros, administrativos europeos sobre la cultura africana.

Las nociones de criollización, de hibridismo lingüístico y literario se intensifican con el movimiento de la negritud. Aimé Césaire pide una criollización de las lenguas europeas para reflejar con exactitud la realidad africana que el autor quiere presentar. La literatura será portal de desemantización, resemantizaciones y de préstamos de palabras de las lenguas negroafricanas en la construcción de las frases en lenguas europeas. Primero considerada como signo de la lucha contra el imperialismo lingüístico, esa apropiación de las lenguas europeas por los autores subsaharianos concurrirá al enriquecimiento de dichos idiomas. Además permiten constituir una autenticidad creativa negroafricana que culmina con Ahmadou Kourouma. El escritor no sólo se permite incluir palabras y textos orales en malinké en sus novelas, sino que presta de los griots distintas técnicas: la enumeración de la genealogía de los personajes para dar más importancia a sus personalidades, la asociación de los reyes con animales, la mistificación de eventos....

Para muchos autores negroafricanos, el hibridismo literario negroafricano nace desde la creación cognitiva. Senghor subrayaba que "j’écris en français, je pense en négro-africain" 19 . Amadou Kourouma en la misma linea menciona : “J'ai pensé en malinké et écrit en français —que j’estime

17. Alfonso De Toro, "Pasajes - Heterotopías - Transculturalidad: estrategias de hibridación en las literaturas latino/ americanas: un acercamiento teórico”, 2005. Disponible a la dirreción http://www.unileipzig.de/ detoro/sonstiges/ Pasajes_Heterotopias_Transculturalidad.pdf> (consulta: 31.10.2014).

18. Esas tres novelas son las primeras publicadas en por africanos en lenguas europeas. Son respectivamente de Ephraim Casely Hayford Ahmadou Mapaté Diagne y Leoncio Evita Enoy.

19. Léopold Sédar Senghor, Liberté I, Seuil, Paris, 1994. 
naturelle - avec la langue classique. J'ai traduit le malinké en français en cassant le français pour retrouver le rythme africain." ${ }^{20}$ Ese hibridismo literario anima las creaciones de los escritores de la diáspora negroafricana actual y permite asentar la producción negroafricana en el marco de las literaturas postcoloniales.

La recuperación de los textos orales por los escritores negroafricanos se presenta como uno de los elementos fundamentes del hibridismo literario negroafricano. Animado por una necesidad de dar a conocer la cultura africana por el mundo y sobre todo su riqueza tradicional oral, muchos autores se dedicaron a recuperar cuentos, leyendas, historias de reyes africanos. Un trabajo de traducción que no excluye la originalidad de las producciones de autores como Hamadou Ampate Bâ o Dadié. Bernard Magnier (2012) hablando del sitio de la oralidad en la literatura negroafricana precisa:

Très tôt, les écrivains africains ont tenu à transmettre la richesse du patrimoine oral du continent. Ainsi, entre les albums aux illustrations séduisantes, parfois stéréotypées, qui présentaient une Afrique estampillée carte postale et les publications savantes aux transcriptions fidèles mais austères des Occidentaux, se sont glissés des recueils de contes, légendes, épopées, chants et textes traditionnels, réunis par les poètes, romanciers et dramaturges. Tous ont tenu, dans les marges de leurs textes ou dans des productions spécifiques, à transcrire et traduire cette part du patrimoine menacée de disparition. Compagnons fabuleux de ces histoires, les animaux, aux pouvoirs anthropomorphes ou non, dans leurs confrontations avec quelques humains ou quelques archétypes (ogresses, marâtres, génies, et autres reines et fous) disent tout à la fois, avec sagesse et malice, l'universalité de la condition humaine et la très grande diversité du Continent. ${ }^{21}$

En general, la oralidad es el elemento fundamental en la construcción de la identidad literaria negroafricana y se puede comprobar su presencia en todas las etapas de la literatura al sur del Sahara. Ese lugar otorgado a la literatura oral constituye el primer nivel diferencial entre la literatura negroafricana y otras formas de arte escrito del mundo. El comparatista podrá analizar la presencia de la literatura oral negroafricana en las producciones dichas modernas en lenguas europeas y sobre todo ver su manifestación. Se podrá estudiar cómo las formas y técnicas literarias propias a la literatura oral integran el texto escrito y forman una nueva dinámica literaria que permite trasmitir las realidades de las sociedades negroafricanas. Otro aspecto de importancia del contacto entre lenguas occidentales, literatura occidental y literatura oral negroafricana es la comparación entre las nuevas formas literarias orales negroafricanas (nuevos cuentos, teatros etc...) y las formas orales tradicionales que han sido transcritos a lo largo de la evolución de la historia negroafricana. Es el caso por ejemplo de los cuentos de Inongo Vi Makomé y de Agnes Agboton que en su casi totalidad no se inspiran en los cuentos orales antiguos del subcontinente negro sino que son nuevas historias de contadores modernos quienes quieren conservar una tradición literaria que ha nutrido su infancia. Estudiar esos nuevos contadores (griot, abuelos o abuelas, Mvet) y sobre todo las técnicas que utilizan para redactar sus epopeyas cuentos y fábulas en comparación a las técnicas tradicionales puede resultar relevante.

Se ha de decir que el hibridismo literario negroafricano es reflejo del hibridismo cultural que ha conocido el subcontinente con la colonización. El impacto del imperialismo europeo en África negra

20. Extracto de una entrevista y citado por Bernard Magnier, Panorama des littératures francophones d'Afrique noire, Institut Français, Paris, 2012. p. 46.

21. ibíd. P.7. 
fue importante y supuso cambios tanto a nivel social, político como religioso. Cuando Ahmadou Mapaté Diagne escribe Les trois volontés de Malick (1920) más allá de elogiar la escuela occidental, la obra es testigo de los cambios y clivajes sociales a los cuales estaba sometido el mundo negroafricano. El protagonista de Diagne se aleja de los oficios tradicionales (herrero, ganadero, agricultor...) para abrazar los nuevos trabajos que ofrece la situación colonial (maestro, traductor, controlador...). A parte de la desobediencia a la tradición, la obra nos presenta un personaje principal que rompe con la religión musulmana dominadora en África del Oeste para abrazar la cultura europea considerada liberal. Obra pionera de la literatura de las costumbres negroafricana, Les trois volontés de Malick daba a conocer la psicosis en la que se sumergía la sociedad africana y de la cual, hoy en día empieza a salir pero a un ritmo muy lento. Otras obras seguirán el mismo camino oponiendo tradición con modernidad, cristianismo e islam con la religión negroafricana (Things fall apart; Chinua Achebe), la cultura occidental y la africana (Sous l'orage; Seydou Badian, Mä̈mouna; Abdoulaye Sadji) la escuela tradicional y coránica con la escuela europea (L’enfant noir; Camara Laye). Ese último antagonismo constituirá la base de L'aventure Ambigüe de Cheick Amidou Kane que al mismo tiempo planteará el problema de identidad que separa las sociedades negroafricanas.

\section{Identidad negroafricana}

Analizar desde una perspectiva comparatista la literatura negroafricana remite también a estudiar sobre una línea diacrónica las modificaciones que conoce la identidad negroafricana desde la colonización hasta hoy. Movimientos como el panafricanismo y el African personality se han encargado de construir las bases de esa nueva identidad negroafricana partiendo de la base de la reclamación de una autenticidad negroafricana frente a la colonización europea.

Los problemas de identidad que ofrecen las sociedades negroafricanas son múltiples. Entre otros tenemos: las divisiones entre conservadores tradicionalistas, la exaltación de una cultura negra o los problemas de pertenencias identitaria. La literatura subsahariana escrita se ha construido al torno de esos problemas identitarios. Por eso las dividimos cuatro etapas: la Négritude y la literatura anticolonial, la post-independentista, la literatura de las desilusiones y las literaturas nacionales y de la diáspora. Un análisis comparado del fondo literario negroafricano podría resaltar esos problemas de identidad. Además, se puede estudiar las relaciones existentes entre identidad negroafricana y literaturas nacionales, étnicas o de la diáspora.

\section{Literaturas nacionales, étnicas y literatura de la diáspora}

Los eventos que surgen tanto en el mundo político como literario negroafricano a partir de los años ochenta ofrecen una visión diferente de la literatura negroafricana. Con las dictaduras, la pérdida progresiva de interés literario sobre el tema de la opresión, la reformulación de las relaciones entre naciones africanas, la identidad nacional empezó a sustituir la identidad africana. Con la Negritud o el Panafricanismo, se había asistido a un escritura representativa que aunque publicado por autores pertenecientes a países diferentes, el hilo conductor era primero una reivindicación de la autenticidad negroafricana, luego la exaltación de la libertad del continente y sobre todo de su arte y la denuncia y crítica de las dictaduras. Pero a partir de los años ochenta, el interés sobre la literatura de las desilusiones, el arte negroafricano en general y la crítica del poder colonial se fue substituyendo por una literatura de costumbres. Las literaturas nacionales toman sus fuentes 
en dicho cambio y reciben ayuda de las editoriales nacionales que vienen a sustituir a grandes editoriales negroafricanas como Présence Africaine.

Se ha de mencionar sin embargo que hablar en un sentido estricto de literatura nacional en África Negra resulta imposible. Natalia Álvaro Méndez ${ }^{22}$ subraya el multiculturalismo estatal como primer freno a la consideración de las literaturas producidas dentro de un mismo espacio político y estatal como literatura nacional. La visión de Álvaro Méndez se explica por el hecho de que en África existen etnias transnacionales. Las fronteras diseñadas durante el congreso de Berlín en 1882 y ajustado a lo largo de la colonización por las metrópolis europeas ayudaron a dividir etnias y distribuirlas entre países. Es el caso por ejemplo por la presencia de la etnia Peul entre catorce países de África del Oeste y África central (de Mauritania hasta Chad). Aunque la adopción de las lenguas europeas (en mayoría para paliar la dificultad de adoptar la lengua de una etnia) en los países africanos recién soberanos ayudó solucionar el problema de la comunicación entre las distintas etnias que conviven sobre un mismo territorio político, no llegó a modificar los sentimientos de pertenencia étnica. Hoy en día, algunos disturbios políticos y guerras civiles negroafricanas están asociados a ese problema étnico ${ }^{23}$

Un análisis de la concepción de las nociones de etnia, nación y África por los autores y confinados en las obras negroafricanas podrá interesar el comparatista. Se podrá estudiar las trascendencias de los autores de la etnia a la nación y sobre todo la recepción del escritor fuera de su etnia. Uno de los ejemplos sería el análisis de la recepción de la literatura anglófona camerunesa en la parte francófona del país y vice-versa. Si asumimos que el autor negroafricano se inspirar de los problemas de su entorno, de su procedencia étnica y de las la realidades comunes al conjunto tradicional al que pertenece, el comparatista compila obras que van más allá de las etnias para ver cómo llegan a representar las realidades nacionales.

La literatura de la "migritude" o de la "diasporization" negroafricana podría ser categorizada como producción que desborda los contextos étnicos para interesarse —en este caso- en la condición negroafricana. Desde el exilio voluntario o involuntario, los autores negroafricanos han producido una importante creación literaria que llama a un análisis particular y una crítica singular. La reducción e integración de los conceptos de etnias y nación en el de transnacionalidad que se nota en las obras de la diáspora negroafricana ha ido incrementado con el distanciamiento físico del autor de su nación. Dos temas guían la producción negroafricana de la inmigración: la búsqueda de un identidad (amplificada por el exilio) y la crítica de las costumbres, las políticas y las tradiciones negroafricanas. El estudio comparatista aquí se podrá focalizar sobre la visión del mundo de los autores para desvelar su comprensión de las culturas a las que pertenecen. Se podrá analizar también la concepción y comprensión de la inmigración negroafricana a partir de las obras.

Considerando lo que hemos expuesto en este apartado y apoyándonos sobre los estudios de Jacques Chevrier ${ }^{24}$ y Álvaro Méndez ${ }^{25}$, pensamos que las líneas siguientes pueden ser del interés del comparatista intrigado por la literatura negroafricana:

22. Natalia Álvaro Méndez (2012), "Africanismo y literatura comparada" en Metodologias comparatistas y literatura comparada, Clásico y Yekinson, Madrid, 2012.

23. Entre otros conflictos bélicos ligados con las etnias tenemos el Genocidio de Ruanda de 1994. El conflicto ocasionó la muerte de ochocientas personas y oponía los Hutus y los Tutsis; dos etnias mayoritarias de Ruanda.

24. Jacques Chevrier, "Las literaturas africanas en campo de la investigación comparada" en Compendio de literatura comparada (dir: Pierre Brunel; Yves Chevrel), Siglo Veintiuno, Madrid, 1994.

25. Op.cit. 
- La relación entre la literatura oral y la literatura escrita en lenguas europeas en África Subsahariana.

- Las influencias de los autores occidentales sobre la producción literaria negroafricana. Un estudio de las influencias de las corrientes literarias y sociales que marcaron la Europa del siglo XX sobre la sociedad africana y su literatura se ofrece como otra línea de investigación.

- El hibridismo cultural que implica el hibridismo literario se ofrece como unas de las vías de estudio. Se podrá analizar y comparar cómo las formas estéticas y estilísticas occidentales son recibidas e integradas en las producciones negroafricanas. Sobre una base sociolingüística, se podrá ver cómo los contactos entre las lenguas europeas y negroafricanas han producido nuevas formas de expresión que enriquecen ambos campos. Además, con el pidgin del inglés, el camfranglais y otras lenguas urbanas, se podrá ver cómo el contacto entre las lenguas han creado otras. El comparatista podrá estudiar las obras negroafricanas a la búsqueda de la presencia de dichas lenguas nuevas.

- El replanteamiento del concepto de literatura nacional en África debido al estudio detallado de las etnias transnacionales, la multietnicidad de los países y la noción de globalización actual.

- La comparación de los autores negroafricanos con otros procedentes de literaturas del mundo. Se tratará de establecer las semejanzas y las diferencias entre la literatura postcolonial negroafricana con las demás como por ejemplo la literatura magrebí postcolonial o por ejemplo la literatura sudamericana. Destacábamos más arriba la importancia de hacer comparaciones entre los cuentos y epopeyas negroafricanos con los de Rusia por ejemplo para resaltar los paralelismos existentes.

- La interconexión entre las distintas etapas de la literatura negroafricana. Por ejemplo ver cuáles son las influencias de la Negritud, de la literatura de las desilusiones sobre la literatura de la diáspora negroafricana actual.

El comparatista interesado en la literatura negroafricana podrá analizar el contenido textual y temático de las obras. En ese caso, se podrá por ejemplo analizar las novelas de Senghor para ver cómo progresivamente y según su implicación en la política, sus escritos evolucionan del compromiso social a preceptos políticos y posteriormente a una centralización en prescripciones filosóficas y sociológicas. Aquí, las metodologías diseñadas por Pierre Brunel sobre la búsqueda del "extranjero en el texto" ${ }^{26}$, los grados de comparatismo de Haddad Wotling y Francis Claudon ${ }^{27}$, los componentes de una metodología comparatista de Manfred Schmeling (1984) ${ }^{28}$ o la doble prescripción de Van Tieghem (1931) ${ }^{29}$ ayudarán el comparatista a acercarse a las obras negroafricanas e ir más allá de la africanidad de las obras tal como lo propone Jacques Chevrier.

Natalia Álvaro Méndez sugiere como líneas de investigación, el estudio de los elementos estéticos procedentes de la influencia externa y oral. En ese caso, se podrá analizar a partir de las obras y sobre todo de las técnicas de escritura, la evolución de los géneros literarios en la literatura subsahariana, las interacciones entre literatura oral y literatura escrita o los estudios de las influencias de los autores negroafricanos.

26. Pierre Brunel, Yves Chevrel, "El hecho comparatista", Compendio de literatura comparada; Siglo Veintiuno, Madrid, 1994.

27. Francis Claudon, Karen Haddad Wotling, Précis de Littérature comparée, Armand Colins, Paris, 2008.

28. Manfred Schmeling, Teoría y praxis de la literatura comparada, Editorial Alfa, Barcelona, 1984.

29. Paul Van Tieghem, La littérature comparée, Armand Colin, Paris, 1931. 


\section{Referencias bibliográficas}

ÁLVARO MÉNDEZ, N., "Africanismo y literatura comparada”, Metodologias comparatistas y literatura comparada, Clásico y Yekinson, Madrid, 2012.

AULLÓN DE HARO, P., "Epistemología de la teoría y crítica literatura" in Teoría de la crítica literaria, Trotta, Madrid, 1994.

CHEVREL, Y., La littérature comparée, Presses Universitaires de France, Paris, 1989.

CHEVRIER, J., "Las literaturas africanas en campo de la investigación comparada", Compendio de literatura comparada (dir: Pierre Brunel; Yves Chevrel), Siglo Veintiuno, Madrid, 1994.

CLAUDON, F., HADDAD WOTLING, K., Précis de Littérature comparée, Armand Colin, Paris, 2008.

DETORO, A., Pasajes - Heterotopias - Transculturalidad: estrategias de hibridación en las literaturas latino/americanas: un acercamiento teórico, 2005. Disponible en : www.unileipzig.de/-detoro/ sonstiges/Pasajes_Heterotopias_Transculturalidad.pdf MAGNIER, B., Panorama des littératures francophones d'Afrique noire, Institut Français, Paris, 2012.

GIL-ALBARELLOS PÉREZ-PEDRERO, S., Introducción a la literatura comparada, Valladolid, Universidad de Valladolid, Secretariado de Publicaciones e Intercambio Editorial, Valladolid, 2006.

GUYARD, M.F., la Littérature comparée, Presses Universitaires de France, Paris, 1978.

MCLEOD, J., Beginning postcolonialism, Manchester University Press, Manchester, 2000.

MOURA, J.M., "Postcolonialisme et comparatisme" en Vox poética, 2006, consultable en línea en : http://www.vox-poetica.com/sflgc/biblio/moura.html

SCHMELING, M., Teoría y praxis de la literatura comparada, Editorial Alfa, Barcelona, 1984.

SÉDAR SENGHOR, L., Liberté I, Seuil, Paris, 1994.

VAN TIEGHEM, P., La littérature comparée, Armand Colin, Paris, 1931.

WELLECK, R., "The name and nature of comparative literature" en Discrimination, further concepts of criticism, Yale University Press, New Haven and London, 1970. 